\title{
SOME REMARKS ON THE HISTORICAL DEVELOP- MENT AND THE FUTURE PROSPECTS OF THE DIFFERENTIAL GEOMETRY OF PLANE CURVES.*
}

BY PROFESSOR E. J. WILCZYNSKI.

Probably the most fundamental characteristic of the human mind is its hatred for contradictions. All of our thinking is fundamentally influenced by this dislike; and the rôle of the mathematician, in his relation to reality, may be described in a fairly adequate manner by saying that it is his business to remove all contradictions from our discussions and, by gradually extending the scope of these discussions, to show that the world as a whole is thinkable.

To justify the validity, in the purely mathematical sense, of any construction of the intellect, absence of contradictions is necessary and sufficient. But the mere absence of contradictions from a realm of thought does not necessarily give it that essential artistic and harmonious one-ness which leads us to think of it as a unit. A peculiarity of the human mind, almost as important as its hatred for contradictions, is its dislike for sudden and frequent changes in the point of view. Thus, quite apart from the obvious practical difficulties of studying plants and stars and souls at the same time, the mind for the sake of its own peace and convenience, following its desire to move along a straight line, has divided knowledge into compartments, and refuses to think of more than one of these compartments at the same time. This procedure does not disturb in the least the profound conviction, present I believe in all thinkers, that at some future time from some other higher point of view the separateness of these compartments will be abolished. Indeed, we cannot help but think that a thoroughgoing unification of each separate realm is the best possible preparation for an ultimate and complete generalization which shall include the whole.

It is my purpose to-day to try and show you how one funda-

* Address of the retiring chairman of the Chicago Section of the American Mathematical Society, read at Columbus, Ohio, December 30, 1915. 
mental idea has dominated and still dominates in the realm of differential geometry. I shall confine my discussion to the differential geometry of plane curves primarily on account of the comparative ease with which we can visualize configurations in the plane. But it is a notable fact that even in this limited domain so many problems have remained untouched, problems which it is easy to formulate and not difficult to solve. Some of the notions which I shall discuss, although conceived in admirable fashion nearly a century ago, have remained practically unnoticed. You would look for them in vain in any of our modern treatises, although these contain many other things of far greater difficulty and of much less interest. In fact, so completely have these ideas been neglected, that most mathematicians are probably under the impression that the differential geometry of plane curves is a very much restricted and uninteresting field not at all adapted for further research. Nevertheless it is true that scarcely one of the notions which will arise in our discussion to-day has been studied as fully as it deserves, while most of them have not as yet received any consideration whatever.

The notion of a general plane curve is at bottom identical with the general notion of function. It is obviously impossible to prove, by documentary evidence, that the ancient geometers did not possess the idea of a general curve. But we may assert, I think, that the available evidence indicates that the ancients knew how to deal only with very special curves such as straight lines, circles, conics, and a few other curves, including certain spirals. The relation between the notions " function" and " curve" only became evident in the seventeenth century of our era, when Descartes and Fermat had laid the foundations of analytic geometry, and it was the recognition of this relation which brought the notion of a general curve into the consciousness of mathematicians everywhere. But analytic geometry did more than merely formulate the notion of a general curve; it also provided a method for its investigation. If the first fruit of this union between analysis and geometry seemed to be of profit primarily for geometry by providing it with a new and limitless field for research, it soon became apparent that the union was to be profitable for analysis also. For the geometric problems which arose in this connection, such as the construction of a tangent to a curve with a given equation, the determination of the length of a 
given arc of the curve, the calculation of areas bounded wholly or partly by curved lines, led inevitably toward the invention of the calculus. In fact, the method employed by Fermat, for instance, for determining the tangent of a curve really involved the essential processes of the differential calculus. This method is very simple and consists in formulating the definition of a tangent as follows. Take a point $P$ on the curve, the point whose tangent we wish to draw. Join $P$ to a second point $Q$ which is also on the curve, and let us seek the limiting position which the line joining $P$ to $Q$ approaches, when $Q$, moving always along the curve, approaches $P$ as a limit. This limiting position of the secant $P Q$ is called the tangent.

Of course it was evident that the tangent would pass through $P$; the only problem was that of finding the direction of the tangent. The calculus, as developed by Newton and Leibniz, made it an easy matter to translate Fermat's definition of a tangent into the language of analysis and then actually to determine, by calculation, the direction of the tangent for a very extensive class of curves. Thus the problem of tangents could be regarded as solved. The normal could then be defined as a line perpendicular to the tangent at the point of contact, and its determination offered no further difficulty. It is worthy of remark, however, that one of the methods proposed by Descartes determines the normal first and the tangent afterward. We shall amplify this remark, a little later.

The analytic formulation of the notion of radius of curvature soon followed. Let us take three points $P, Q$, and $R$ on a curve and let us pass a circle through these points. As $Q$ and $R$ move along the curve, approaching $P$ as a limit, this circle will in general approach a limiting position which is called the circle of curvature. Its radius is called the radius of curvature, and its center the center of curvature. The familiar formula for the radius of curvature was published, apparently for the first time, in Newton's "Methodus Fluxionum" of 1736, although the notion itself was much older and was applied with great success by Huygens as we shall see immediately.

If the curve under consideration is itself a circle, the circle of curvature of every one of its points is of course that same circle. But if the original curve is not a circle, each of its points will have in general a different circle of curvature and the problem arises to find the locus of the centers of all of these 
circles. This locus, called the evolute of the given curve, was first considered by Huygens, without of course making use of the notations of the calculus, in his "Horologium Oscillatorium" published in 1673. Huygens saw that the normals of the original curve would be the tangents of the evolute, and that the original curve could be regarded as the locus of the endpoint of a string which was being gradually unwound from the evolute. This showed him that the difference between the lengths of two radii of curvature of the original curve was equal to the length of the corresponding arc of the evolute. Since the length of the string could be changed, it became apparent that, while every curve has only one evolute, it is itself the evolute of infinitely many other curves which are called its involutes. These involutes moreover are clearly the orthogonal trajectories of the tangents of the given curve.

For Huygens these notions were of great importance as applied to the special case of the cycloid, a curve first considered by Galileo. For he had recognized the isochronous property of the cycloid and had therefore shown that the cycloid was the most desirable curve for the oscillations of a pendulum. The problem now arose to devise a method which would compel the oscillations of a pendulum to take place along a cycloid. Huygens observed in this connection that a weight attached to a string would describe an arc of a cycloid if the string were attached in such a way as to cause it to wind and unwind along checks which had been given the form of an evolute of a cycloid. In fact it was this problem which led Huygens to his general theory of evolutes. He now found the remarkable theorem that the evolute of a cycloid is an equal cycloid, a theorem which must have appeared to him as a beautiful manifestation of the divine harmony of geometry.

The tangent may be regarded as a first approximation to the given curve in the neighborhood of one of its points. In modern terminology we may say that the tangent serves as a geometric image for the first derivative $f^{\prime}(x)$ of the function $f(x)$, whose graph is the curve under consideration. The circle of curvature may be regarded as a second approximation to the curve or as a geometric image of the second derivative of $f(x)$. For a long time no attempt was made to find a geometric image for the third derivative. In 1841, however, Abel Transon published his beautiful "Recherches sur la courbure des lignes et des surfaces," in which he takes not only 
this step but also the next, by devising appropriate geometric images both for the third and fourth order derivatives.

Transon first introduces the new notion which he calls "déviation" and which has been translated by the term "aberrancy" in the very few places where any notice has been taken of Transon's work. This notion is as follows. Let $P$ be a point on a curve and let a chord be drawn parallel to the tangent at $P$ and very close to this tangent. Let $Q$ and $R$ be the points (in the neighborhood of $P$ ) in which this chord intersects the curve, and let $L$ be the middle point of $Q R$. As $Q R$ approaches the tangent at $P$ as a limit, the line $P L$ will in general approach a limiting position called the axis of aberrancy. Let $\delta$ be the angle which the axis of aberrancy makes with the normal. The tangent of this angle is given by the formula

$$
\tan \delta=\frac{d y}{d x}-\frac{\left[1+(d y / d x)^{2}\right]}{3\left(d^{2} y / d x^{2}\right)^{2}} \frac{d^{3} y}{d x^{3}} .
$$

and Transon calls $\tan \delta$ the aberrancy of the curve at the point $P$. It would obviously be equal to zero at any point of a circle.

From familiar properties of conic sections it follows at once that the axis of aberrancy at any point of a conic is the line which joins this point to the center of the conic. Let us observe further that the expression (1) for the aberrancy contains only the first, second, and third derivatives of $y=f(x)$, so that the aberrancy is indeed adapted for the purpose of visualizing the third derivative.

The equation of a conic contains five essential constants, and a conic is therefore determined by five of its points. The condition that one curve shall have third order contact with another, may be expressed by saying that four of the points of intersection of the two curves coincide. Consequently there exists a one-parameter family (a pencil) of conics each of which has third order contact with the given curve at the given point $P$. All of these conics pass through $P$; they all have the same tangent, the same circle of curvature, and the same axis of aberrancy at $P$. Among these conics there will be one and only one parabola; let us call it the osculating parabola. Since, as we have just seen, the axis of aberrancy of the point $P$ of our given curve is also the axis of aberrancy for each of the conics which has third order contact with our curve at $P$, and since the axis of aberrancy for a point of a conic passes 
through the center of the conic, and since finally the center of a parabola is at infinity, we see that the axis of the osculating parabola is parallel to the axis of aberrancy.

To locate the axis of the osculating parabola completely we have, in addition to the remark just made, the following simple construction also due to Transon. Let $D$ be the orthogonal projection of the center of curvature $C$ upon the axis of aberrancy, and let $E$ be the orthogonal projection of $D$ upon the normal. Then the axis of the osculating parabola will pass through $E$. Since it must also be parallel to the axis of aberrancy we may now regard the axis of the osculating parabola as known.

The focus of this parabola must clearly be on a line through the point $P$ which makes with the normal at $P$ an angle equal to that made with this same normal by the axis of aberrancy. Thus the focus will be the intersection of this line with the axis. The directrix will of course be perpendicular to the axis. It will also pass through a point on the normal on the convex side of the curve whose distance from $P$ is equal to half the radius of curvature. This last result is obtained by Transon as a corollary from a more general theorem. He considers the one-parameter family of parabolas, each of which has second order contact with the given curve at $P$. The directrices of all of these parabolas pass through the point just described, and the locus of their foci is a circle whose diameter is that half of the radius of curvature which terminates at $P$.

Among the conics which have third order contact with the given curve at $P$, there will be one for which the order of contact rises to the fourth order at least. This is the osculating conic of the point $P$ and may be regarded as having five consecutive points in common with the curve. In common with all of the conics having third order contact with the given curve at $P$, it has its center on the axis of aberrancy, and the position of the center on the axis of aberrancy may therefore be regarded as a geometric equivalent for the value which the fourth derivative of $y=f(x)$ assumes at the point $P$. The properties which I have mentioned suffice to determine the osculating conic, but Transon develops some further theorems which facilitate its construction very considerably. I shall quote some of these on account of their great geometric interest. 
These theorems are again concerned with the pencil of conics which have third order contact with the given curve at $P$. Transon finds that the axes of these conics envelop a parabola, whose directrix is the axis of aberrancy, and whose focus is the orthogonal projection of the center of curvature upon the line which joins $P$ to the focus of the osculating parabola. This latter line is very easy to construct since, as we have seen, the normal bisects the angle between this line and the axis of aberrancy. The auxiliary parabola of Transon, I should like to add, also touches the lines which are tangent and normal to our original curve at $P$. As a consequence of this theorem of Transon's the directions of the principal axes of the osculating conic are obtained by drawing the two tangents from the center of the osculating conic to the auxiliary parabola.

The following property, not mentioned by Transon, is also of interest. Among the conics which have third order or fourpointic contact with the given curve at $P$, there will be infinitely many hyperbolas and ellipses, but in general no circle. This indicates the existence, in this family of conics, of a unique ellipse of minimum eccentricity. As the center of such a four-pointic conic moves along the axis of aberrancy on the concave side of the curve, starting from $P$, the eccentricity $e$ of the corresponding ellipse will decrease continuously from unity toward the minimum value $e_{1}$, and will then increase, approaching the limit 1 as the center recedes beyond bound. Thus every value of $e$, between the minimum value $e_{1}$ and unity, will be attained twice. It is not difficult to show that the center $O$ of the ellipse of minimum eccentricity and the centers, $O_{1}$ and $O_{2}$, of any two of these ellipses which have the same eccentricity are so related that $P O$ is the geometric mean of $O P_{1}$ and $O P_{2}$. In general this four-pointic ellipse of minimum eccentricity is quite distinct from the osculating conic. It will coincide with the osculating conic however if and only if the original curve is a logarithmic spiral. Moreover, the eccentricity of the four-pointic ellipse of minimum eccentricity will in general change as $P$ moves along the given curve. It will be constant if and only if the given curve is a logarithmic spiral.

We have seen how many interesting questions present themselves when we attempt to explore the relations between these various configurations all of which are determined by 
the properties of a given curve in a single one of its points. But each of these configurations gives rise to new problems if we think of the point $P$ as moving along the given curve. Thus, in the simplest case, already mentioned, the locus of the centers of curvature defines a new curve, which is at the same time the envelope of the normals which is called the evolute. In similar fashion we may investigate the envelope of the axes of aberrancy of a given curve. This envelope is at the same time the locus of the centers of its osculating conics. We may also study the envelopes of the axes and directrices of the osculating parabolas, the loci of their foci and vertices, and it is easy to formulate corresponding problems for the osculating conic and for the four-pointic ellipse of minimum eccentricity. Only a mere beginning of such a theory is available at present and most of this is due to Cesàro who made use of some of these notions in his "Lezioni di Geometria intrinseca," with the main emphasis however not upon the general theory but upon the application to certain well-known simple curves.

But I wish to call your attention primarily to a general method which enables us to push still farther the investigation of the properties of a curve in the vicinity of one of its points. The details which I have presented will serve to make clear the following general notions. Let us consider an equation of the form

$$
\varphi\left(x, y ; a_{1}, a_{2}, \cdots, a_{n}\right)=0
$$

which involves the coordinates $(x, y)$ of a point and $n$ essential constants $a_{1}, a_{2}, \cdots, a_{n}$. For every set of values $a_{1}, a_{2}, \cdots, a_{n}$ this equation represents a curve. All of the curves represented by such an equation are said to form an $n$-parameter family. The condition that a curve of this family should pass through a given point gives rise to one relation between the parameters $a_{1}, \cdots, a_{n}$. Thus, in general, one curve of the family, or a finite number of such curves, is determined by the condition that the curve shall pass through $n$ given points. If the parameters enter the equation (2) linearly, as is most frequently the case, there will be just one such curve passing through $n$ given points, if we leave aside certain exceptional cases.

If then we take $n$ points on any given curve, there will exist in general a unique curve of the $n$-parameter family (2) 
which passes through them, and this curve will ordinarily approach a definite curve of the $n$-parameter family as a limit, if the $n$ given points approach coincidence. The resulting limit curve will be said to osculate the given curve at the given point, in as much as it will have closer contact ( $n$-pointic or $(n-1)$ th order contact) with the given curve at the given point than any other curve of the class defined by (2). To illustrate this notion we observe that the straight lines of the plane form a two-parameter family and that the tangent is the osculating straight line; that the circles form a threeparameter family and that the circle of curvature is the osculating circle. Moreover we have already made use of this terminology in our discussion of the osculating parabola and the osculating conic.

But our previous discussion shows quite clearly that, besides the osculating curves of a given class, those curves of the class are also of very great interest for which the order of contact falls short of the maximum by a single unit. Let us speak of such curves as penosculants. Evidently, from what has been said, the locus of the centers of the penosculating circles of a given point of a curve is the corresponding normal. In fact it was by means of this remark, to which we have already alluded very briefly, that Descartes defined the normal and thus indirectly solved the problem of tangents. Again we have seen that the locus of the foci of the penosculating parabolas is a circle whose diameter is equal to half the radius of curvature, and that the locus of the centers of the penosculating conics is the axis of aberrancy. Several others of the theorems which we have mentioned may be expressed more compactly by the help of this new terminology.

After this preliminary discussion, let us inquire what classes of osculants and penosculants ought to be introduced for the purpose of providing geometric interpretations for the derivatives of order higher than the fourth. For we may regard the theory outlined so far as exhaustive for the first four orders.

The general curve of the third order contains nine essential constants. Therefore the osculating cubic has nine consecutive points in common with the given curve at $P$. The penosculating cubics have eight consecutive points in common with the given curve at $P$ and form a pencil. One of the cubics of this pencil is of special interest because it has a double point at $P$. Of course one of its double point tangents touches the 
given curve at $P$; the other one crosses the curve at a nonvanishing angle. That branch of the penosculating nodal cubic which actually touches the given curve has sevenpointic or sixth order contact with it, and we may therefore use this cubic as a geometric image for the sixth derivative. The osculating cubic may of course be regarded as a representative of the eighth derivative.

The osculating cubic and the penosculating cubics were introduced by Halphen in his thesis on differential invariants in 1876. Halphen's interest was centered primarily upon the following feature of this situation. All of the penosculating cubics have, besides the eight points of intersection which are concentrated at $P$, a ninth point of intersection which I have called the Halphen point. Now it may happen that this ninth point also coincides with $P$, in which case $P$ is called a coincidence point. This will happen if a certain projective differential invariant (in my notation the invariant $\theta_{8}$ ) vanishes, and Halphen made use of these geometric notions for the purpose of calculating this invariant. He also showed that a curve may be composed entirely of coincidence points and that all such curves may be obtained from a certain logarithmic spiral by projective transformation.

We have now obtained representative osculants for contact of the first four orders and for orders six and eight. It remains to fill the gap for orders five and seven. In order to do this, we may consider special kinds of cubics distinguished by fundamental metric properties, just as the gap between the osculating straight line and the osculating conic was filled by means of osculating curves of order two distinguished by metric properties, namely by the osculating circle and parabola.

A parabola is a conic which touches the line at infinity. We may define a parabolic cubic to be a cubic which touches the line at infinity. Such a cubic contains eight arbitrary constants indicating the existence of osculating parabolic cubics to represent the seventh derivative. These cubics are not however necessarily the best adapted for this purpose. There are other cubics which accomplish as much and which are more easily accessible, as for instance that particular one of the penosculating cubics whose asymptote is parallel to the normal.

A circle may be defined as a conic which contains the socalled circular points at infinity. I shall not attempt to explain 
this notion which is familiar to all mathematicians. A cubic which contains the circular points is called a circular cubic. The general equation of such a cubic contains seven arbitrary constants, indicating the existence of an osculating circular cubic which has seven-pointic or sixth order contact with the given curve, and which will probably for most purposes be a better representative of the sixth derivative than the one mentioned before.

The penosculating circular cubics of the point $P$ all have six consecutive points in common with the given curve at $P$. Besides they intersect each other in the two circular points at infinity. The determination of their ninth point of intersection and the condition for its coincidence with $P$ offer problems which are strictly analogous to the corresponding problems involving the Halphen point. Among the penosculating circular cubics there is one which has a double point at $P$. There is also one whose real asymptote is parallel to the normal. This latter curve may serve as an image for the derivative of the fifth order. But of course there are other cubics, both circular and non-circular, which may serve as well.

We may continue in this way to build up a theory of osculating algebraic curves of gradually increasing order, the osculants of each order being classified further according to the number of times that they pass through the circular points and the number of their asymptotes which are parallel to the normal. However I shall refrain from any further detailed exposition in this direction, only stopping to say that the analytic difficulties involved in actually determining the equations of these osculants up to and including the osculating cubic are far from insuperable. In fact, I actually have most of these equations at my disposal and they are much simpler than one might expect.

The penosculating nodal cubic is of fundamental importance in projective geometry. The following remarks may serve to make this clear. By introducing a suitably chosen system of homogeneous coordinates and denoting the properly chosen ratios of these homogeneous coordinates by $X$ and $Y$, the equation of any curve, if it is neither a straight line nor a conic, may be expanded in the form

$$
Y=\frac{1}{2} X^{2}+X^{5}+A_{7} X^{7}+\cdots
$$


in the vicinity of any one of its ordinary points. In this expansion $A_{7}$ and all of the remaining coefficients will be absolute projective invariants. The simplicity of this expansion, and the uniqueness of its form, make it evident that the system of coordinates, to which this expansion corresponds, must be of peculiar importance. But this system of coordinates is determined entirely by the properties of the penosculating nodal cubic. The triangle of reference has for two of its sides the double point tangents of this cubic and for its third side the line upon which lie its three points of inflection. Incidentally we may remark here that the relation of the simple quintic curve

$$
Y=\frac{1}{2} X^{2}+X^{5}
$$

to the given curve is worthy of notice.

The fundamental importance of the penosculating nodal cubic also appears when we attempt to interpret the simplest of all projective integral invariants. This integral corresponds so nearly to the notion of length of arc, which is the invariant integral of lowest order in the metric theory, as to justify the prediction that it will be found to be of the greatest importance in future developments of the projective theory. Thus, for instance, this integral enables us to formulate at once a notion which generalizes, in the sense of projective geometry, Cesàro's intrinsic equation of a curve.

We have discussed osculating and penosculating curves of many different kinds, all of which however were algebraic. Transon also mentions the availability of the notion of an osculating logarithmic spiral, and in his projective theory Halphen makes use of the notion of an osculating anharmonic curve. The logarithmic spiral and the general anharmonic curve are transcendental curves to be sure, but they belong to a particularly simple type of transcendental curves. In fact, most of these curves are so closely related to algebraic curves that Leibniz thought it inadvisable to speak of them as transcendental, and invented a special name for them, calling them interscendental curves. So far as I am aware, no other curves, except those mentioned, have ever been used as osculants in the theory of plane curves.

Each of the osculants and penosculants which we have introduced has a function to perform which may be illuminated by an aphorism; the osculant is the microscope of the geometer. 
Thus, to the naked eye the courses of two curves, which at a common point have the same tangent and the same radius of curvature, are in the vicinity of that point so nearly identical as to make them appear indistinguishable. The introduction of the notions of axis of aberrancy and osculating parabola serves to magnify the differences between the two curves in such a way as to enable us to distinguish between them. Again, if the two curves also have their osculating parabolas in common, we may judge of their divergence by means of their osculating conics. Thus the notion of osculant serves the differential geometer for the same purpose as does the microscope in the laboratory of the biologist. It magnifies the infinitesimal differences between two different curves sufficiently to cause them to make an emphatic impression upon the mind.

Thus the notions, osculant and penosculant, are the fundamental concepts of differential geometry. The systematic investigation of the magnitudes, loci and envelopes determined by the various classes of osculants and penosculants and the relations which exist between them makes up the whole subject matter of differential geometry. Differential properties of a general curve are merely integral properties of its osculants and penosculants.

The University of Chicago, December, 1915.

\section{A CERTAIN SYSTEM OF LINEAR PARTIAL DIF- FERENTIAL EQUATIONS.}

BY DR. H. BATEMAN.

(Read before the American Mathematical Society, February 26, 1916.)

1. IT is known that if a function $V\left(x_{1}, y_{1}, z_{1}, t_{1} ; x_{2}, y_{2}, z_{2}, t_{2}\right.$; $\left.\cdots ; x_{n}, y_{n}, z_{n}, t_{n}\right)$ satisfies the system of $\frac{1}{2} n(n+1)$ partial differential equations*

(1) $\frac{\partial^{2} V}{\partial x_{p} \partial x_{q}}+\frac{\partial^{2} V}{\partial y_{p} \partial y_{q}}+\frac{\partial^{2} V}{\partial z_{p} \partial z_{q}}=\frac{\partial^{2} V}{\partial t_{p} \partial t_{q}} \quad(p, q=1,2, \cdots, n)$

it becomes a solution of the reduced system of $\frac{1}{2}(n-1) n$

* See for instance H. Bateman, Messenger of Mathematics, March, 1914, p. 164. 